\title{
Probing Phonon and Infrared-Plasmons in Nanoscale Interfaces
}

MJ Lagos ${ }^{1,2 *}$, U Hohenester ${ }^{3}$, A Trugler ${ }^{3}$, V Amarasinghe ${ }^{4}$, LC Feldman ${ }^{4}$, PE Batson ${ }^{4}$ and GA Botton ${ }^{1,2}$

${ }^{1 .}$ Department of Materials Science and Engineering, McMaster University, Hamilton, Ontario, Canada.

2. Canadian Centre for Electron Microscopy, McMaster University, Hamilton, Ontario, Canada.

3. Department of Physics, University of Graz, Graz, Austria.

4. Department of Physics and Astronomy, Rutgers University, Piscataway, New Jersey, USA.

*Corresponding author: mjlagos@mcmaster.ca

Recent developments of highly monochromatic atom-wide electron beams have held promise to allow exploration of phonon excitations in nanomaterials with high spatial resolution. The experimental data, however, can present enormous interpretation challenges, requiring a much deeper understanding of elementary excitations (plasmons, phonons) sustained in finite-size objects, probe screening effects, scattering $f$-sum rules, symmetry selection rules, etc. In this work, we present a study of the phonon and plasmon response in a complex semiconductor/insulator/metal $\left(\mathrm{SiC} / \mathrm{SiO}_{2} / \mathrm{Al}\right)$ trilayer system. In particular, we focus on the interfaces phonon modes between the semiconductor/insulator materials and on the role of the polariton plasmon modes of metallic nanostructures in the scattering response.

We used a Nion UltraStem equipped with a monochromator to study the phonon and plasmon response in the infrared range using a $\sim 1.5-2 \AA$ probe with an energy spread of $\sim 9 \mathrm{meV}$, at $60 \mathrm{kV}$. As previously demonstrated, the combination of high spatial and energy resolution has allowed the detection of acoustic/optical bulk and surface phonon excitations down to $20-30 \mathrm{meV}$, allowing the nanoscale mapping of phonon scattering [1] and the measurement of local temperature [2] using energy gain/loss phonon scattering [3] in individual nanostructures. In this work, we performed spatiallyresolved studies across the interfaces of a $\mathrm{SiC} / \mathrm{SiO}_{2} / \mathrm{Al}$ trilayer system, finding that the scattering signal is strongly dependent on the probe position (Fig. 1).

When the probe intersects the $\mathrm{SiC}$, most of bulk acoustic and optical longitudinal phonon modes of $\mathrm{SiC}$ $(\sim 43,65,110 \mathrm{meV})$ are excited due to the efficient coupling between the fast electron and the lattice vibrations, including surface phonon polaritons (SPh) at $\sim 104 \mathrm{meV}$. We also found a broad small resonance between $130-150 \mathrm{meV}$ due to the aloof excitation of SPhP modes of the neighbouring $\mathrm{SiO}_{2}$ layer. When the probe impinges on the $\mathrm{SiO}_{2}$ layer, $\mathrm{SPhP}$ 's $(\sim 50,135 \mathrm{meV})$ and a bulk phonon mode $(\sim$ $100 \mathrm{meV})$ from the silica are excited [4]. Also, SPhP's from the $\mathrm{SiC}(\sim 104 \mathrm{meV})$ are excited as a result of interaction of the external excitation with the surface modes during the close approach. Across this $\mathrm{SiC} / \mathrm{SiO}_{2}$ interface, we identified interfaces modes $(\sim 135$ and $97 \mathrm{meV})$ as a result of the hybridization between the modes of the silica and $\mathrm{SiC}$ specimens. Those interface modes extend from the interface and decay towards the interior of both materials. We performed theoretical modelling of the inelastic scattering and we found good agreement with the experimental results. When the probe intersects the $\mathrm{Al}$ layer, a large variety of excitation processes are detected. Those include short-wavelength acoustic phonons near/at the first Brillouin zone $(\sim 40 \mathrm{meV})$ whose excitations require matching large amounts of momentum transfer $\left(>1 \AA^{-1}\right)$ during the inelastic scattering. Also, we detected a $125 \mathrm{meV}$ resonance corresponding to the dipole plasmon mode of the $\mathrm{Al}$ antenna. This IR excitation is imposed by the large size $(\sim \mu \mathrm{m})$ of the antenna [5], which leads to the formation of a plasmon mode with linear (photon-like) dispersion. Higher-order plasmon modes are also detected at higher energies. SPhP's of the $\mathrm{SiO}_{2}$ and $\mathrm{SiC}$ structures are also excited in this probe configuration. 
In addition, it is considered that screening of the probe can cause the dipole vibrational signal to be weak or be nearly absent in some materials, revealing short wavelength vibrational behavior associated with the local structure. In this regard, we evaluated the effects of the screening of each material in the interface system, studying independently $\mathrm{SiO}_{2}$ and $\mathrm{SiC}$ wedges. For probing conditions in the intersecting geometry, coupling between the fast electron and bulk modes is observed. For probing conditions in aloof mode, shielding of the coulomb field of the travelling electron by surface plasmon/phonons might lead to the suppression of excited bulk phonon modes.

In summary, we probed interface phonon modes and IR plasmons of a semiconductor/insulator/metal trilayer system, and evaluated the role of the probe screening in the inelastic scattering at interfaces. We reported infrared plasmon modes in micrometer-sized antennas. Our results extend the understanding of phonon and plasmon polariton excitations in finite-size objects. Studies of the plasmon-phonon coupling in nanostructures are in progress [6].

\section{References:}

[1] MJ Lagos et al., Nature 543 (2017), p. 529.

[2] MJ Lagos and PE Batson, NanoLetters 18 (2018), p. 4556.

[3] MJ Lagos and PE Batson, Microscopy and MicroAnalysis 2017 Conference abstract PDP44 (2017). https://www.microscopy.org/MandM/2017/program/abstracts/PDP-44_Lagos.pdf.

[4] MJ Lagos et al., Microscopy 67 (2018), p. i3.

[5] D Rossouw and GA Botton, Physical Review Letters 110 (2013), p. 066801.

[6] For financial support, M.J.L. and G.A.B. acknowledge the Natural Sciences and Engineering Research Council (NSERC) under a discovery grant; P.E.B. acknowledges support of U.S. Department of Energy, Office of Science, Basic Energy Sciences, under award no. DE-SC0005132

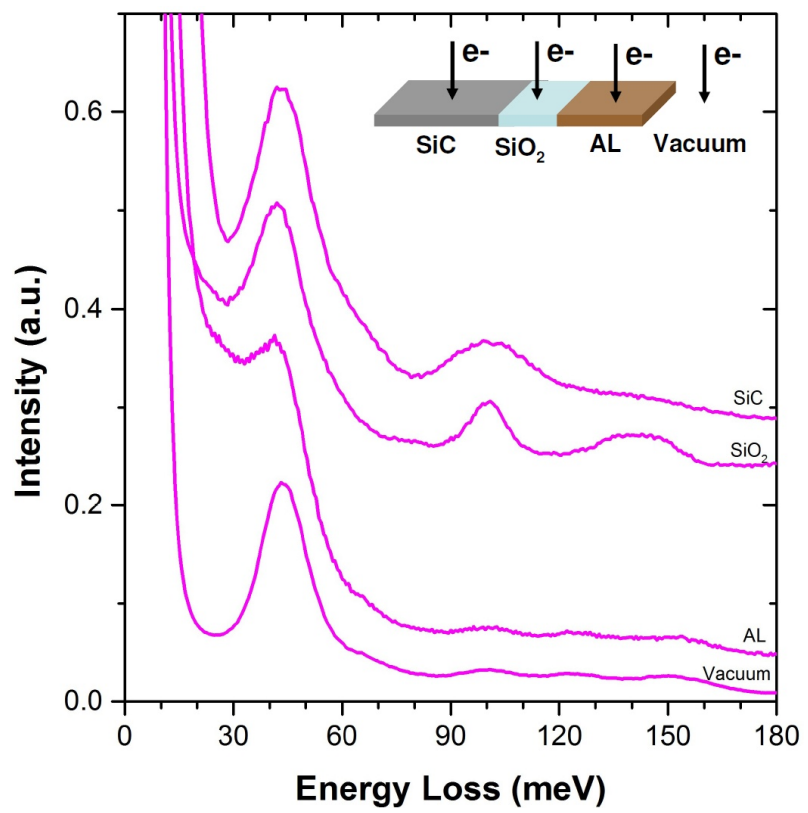

Figure 1. Deconvoluted EELS spectra obtained from a trilayer system $\left(\mathrm{SiC} / \mathrm{SiO}_{2} / \mathrm{Al}\right)$. Inset shows a schematic of the probed system. Note that the inelastic scattering varies as a function of probe position. See text for description. 